\title{
Sonographic abnormalities in pregnancies conceived following IVF with and without preimplantation genetic testing for aneuploidy (PGT-A)
}

\author{
Carrie K. Riestenberg ${ }^{1}$ (1) - Thalia Mok ${ }^{2} \cdot$ Jessica R. Ong ${ }^{2} \cdot$ Lawrence D. Platt $^{2,3} \cdot$ Christina S. Han $^{2,3} \cdot$ Molly M. Quinn ${ }^{1}$
}

Received: 9 August 2020 / Accepted: 7 January 2021 / Published online: 3 February 2021

(C) The Author(s) 2021

\begin{abstract}
Purpose To report the rate of fetal anomalies detected on anatomy ultrasound in pregnant patients who underwent IVF with preimplantation genetic testing for aneuploidy (PGT-A) compared to patients who conceived following IVF with unscreened embryos and age-matched patients with natural conceptions.

Methods Retrospective cohort study at a single maternal-fetal medicine practice. Patients with singleton pregnancies who had a mid-trimester anatomy ultrasound between January 2017 and December 2018 were screened for inclusion. A total of 712 patients who conceived after IVF with or without PGT-A were age-matched with natural conception controls. The primary outcome was the rate of fetal and placental anomalies detected on mid-trimester anatomical survey. Secondary outcomes included the rates of abnormal nuchal translucency (NT), second trimester serum analytes, non-invasive prenatal testing (NIPT), and invasive diagnostic testing.

Result(s) There were no differences in the rate of fetal anomalies in patients who underwent IVF with PGT-A compared to patients who conceived following IVF with unscreened embryos and age-matched patients with natural conceptions. Rate of abnormal NT, high-risk NIPT, and abnormal invasive diagnostic testing were also similar. Patients who conceived after IVF with or without PGT-A had higher rates of abnormal placental ultrasound findings and abnormal second trimester serum analytes compared to natural conception controls.

Conclusion The use of PGT-A was not associated with a difference in risk of fetal anomaly detection on a mid-trimester anatomical survey. The results of this study highlight the importance of improved patient counseling regarding the limitations of PGT-A, and of providing standard prenatal care for pregnancies conceived through ART, regardless of whether PGT-A was performed.
\end{abstract}

Keywords IVF · Preimplantation genetic testing (PGT-A) · Fetal anomalies

Carrie K. Riestenberg

criestenberg@gmail.com

Molly M. Quinn

mquinn@mednet.ucla.edu

1 Division of Reproductive Endocrinology and Infertility, Department of Obstetrics and Gynecology, University of California, Los Angeles, 10833 Le Conte Avenue 27-139 CHS, Los Angeles, CA 90095-1740, USA

2 Division of Maternal-Fetal Medicine, Department of Obstetrics and Gynecology, University of California, Los Angeles, 10833 Le Conte Avenue 27-139 CHS, Los Angeles, CA 90095-1740, USA

3 Center for Fetal Medicine \& Women's Ultrasound, 6310 San Vicente Blvd, Suite 520, Los Angeles, CA 90048, USA

\section{Introduction}

Preimplantation genetic testing for aneuploidy (PGT-A) has been increasingly adopted by in vitro fertilization (IVF) clinics across the USA, with reported use in over $40 \%$ of autologous IVF cycles in 2018 [1]. Providers have turned to PGT-A due to the potential for improved pregnancy and live birth rates on a per-cycle basis [2-5]. However, a recent multicenter randomized control trial found no benefit of PGT-A compared to embryo selection by morphology alone [6].

Ethical concerns pertaining to the rapid uptake of the PGTA technology include safety, cost, and a misunderstanding of the predictive value of PGT-A results. A survey of 300 patients undergoing IVF at a high-volume academic IVF center found that patients often hold misconceptions about the utility 
of PGT-A, with the majority of patients indicating that their primary reason for pursuing PGT-A was to increase the chance of having a "healthy baby," and nearly one-fifth of patients indicating that it was to reduce the risk of congenital anomalies or "birth defects" [7].

Abnormalities of fetal anatomy detected on prenatal ultrasound are reported in approximately $3-4 \%$ of pregnancies [8]. It is estimated that nearly one quarter of congenital anomalies are secondary to a genetic etiology, and 5-10\% result from identifiable environmental or maternal exposures. However, an etiology remains unidentifiable in two-thirds of cases [9, 10]. With a modern ultrasound technology, sensitivity of antenatal ultrasound for the detection of fetal anomalies has significantly improved, with detection rates of approximately 93\% for cleft lip and palate, 75-83\% for abdominal wall defects, and $53 \%$ for selected heart defects [11-13]. Midtrimester ultrasound is a critical point at which the fetal anatomy is evaluated in detail. Abnormalities detected on midtrimester ultrasound influence the management of the remainder of the pregnancy and have been found to be significantly associated with the likelihood of pregnancy termination [14].

Few studies have investigated the rate of congenital anomalies in pregnancies resulting from IVF with PGT-A. Zhang et al. found no overall difference in the rate of congenital anomalies between patients who underwent IVF with or without PGT-A followed by frozen or fresh embryo transfer (ET) [15]. Importantly, this study excluded miscarriages and pregnancy terminations for fetal anomalies and will therefore inherently underestimate the prevalence of anomalies in both groups.

The goal of the present study was to report the rates and specific types of fetal and placental anomalies detected on mid-trimester anatomy ultrasound in patients who underwent IVF with PGT-A compared to patients who conceived following IVF with unscreened embryos and age-matched patients with natural conceptions.

\section{Materials and methods}

\section{Patients}

This was a retrospective cohort study at a single high-volume metropolitan maternal-fetal medicine referral center. All patients with singleton pregnancies who had a mid-trimester anatomy ultrasound between January 1, 2017, and December 31, 2018, were screened for inclusion. The electronic medical record was queried to identify patients with singleton pregnancies conceived with IVF with and without PGT-A during the study period. Multifetal gestations and patients without a mid-trimester anatomy ultrasound were excluded. All anatomical surveys at the center were performed by board-certified maternal-fetal medicine physicians using standardized protocols. One-to-one matching with naturally conceived pregnancies during the same period was subsequently performed based on patient age at the time of anatomy ultrasound. For patients who conceived with IVF using donor egg, the age of the egg donor at the time of retrieval was used for age matching.

The charts were systematically reviewed and the data extracted by three members of the research team (CR, TM, JRO) using a shared protocol. Accurate categorization of pregnancies (IVF, IVF + PGT-A, or natural conception) was confirmed by a chart review. Fetal anomaly data was obtained from the targeted anatomy ultrasound report and categorized as major or minor and by organ system according to the Center for Disease Control and Prevention (CDC) guidelines [16]. Abnormal placental findings noted on anatomy ultrasound including placenta previa, velamentous cord insertion, single umbilical artery, presence of a succenturiate placental lobe, and evidence of placenta accreta were also recorded. Patient demographics, including body mass index (BMI), parity, and maternal comorbidities were extracted. Maternal comorbidities reviewed included hypertensive disorders, gestational and pregestational diabetes, autoimmune disease, and cardiac disorders. The primary outcome was the rate of anomalies detected at the time of the anatomy ultrasound. Secondary outcomes included rate of abnormal nuchal translucency (NT), abnormal second trimester serum analytes, high-risk non-invasive prenatal testing (NIPT), and abnormal invasive diagnostic testing (chorionic villus sampling (CVS) and amniocentesis). NT measurements were performed by sonographers and physicians certified by the NT Quality Review Program. NT was classified as abnormal if the measurement was greater than or equal to $3 \mathrm{~mm}$ [17]. Second trimester serum screen consisted of human chorionic gonadotropin, alpha fetoprotein, inhibin A, and unconjugated estriol [18, 19]. Abnormal second trimester screen was determined by the California Prenatal Screening Program age-based cutoffs for serum analytes. Patients who underwent diagnostic testing via CVS or amniocentesis had genetic testing through different platforms based on indication for testing. The platforms used included karyotype, chromosomal microarray (CMA), and fluorescent in situ hybridization (FISH). Indications for invasive diagnostic testing included abnormal ultrasound finding, genetic abnormality in a prior pregnancy, personal or family history of genetic disorder, and advanced maternal age.

\section{Statistical analysis}

To detect a reduction in ultrasound-detected anomalies from 3 to $1 \%$ (OR 0.33), 768 patients would be needed per group. Our convenience sample of 2 years of ultrasounds performed on patients who had undergone IVF yielded 712 patients. A power calculation indicated that a sample size of 710 patients 
per group would afford $80 \%$ power to detect an OR of 0.27 , or a difference from a baseline rate of ultrasound-detected anomalies of 3 to $0.8 \%$.

Comparison of continuous variables was performed using Student's $t$ test. Categorical variables were compared using the chi-square or fisher's exact test where applicable. Logistic regression was used for multivariate analysis. Statistical significance was set at a $p$ value of $<0.05$ with two-tailed testing. Statistical analyses were performed using STATA version 14 (Stata Corp., College Station, TX).

\section{Ethical approval}

This study was approved by the Institutional Review Board of the University of California, Los Angeles (IRB\# 19-000222).

\section{Results}

A total of 712 patients with singleton pregnancies who conceived with IVF had a mid-trimester ultrasound performed during the study period. Compared to age-matched natural conception controls, patients who conceived after IVF had significantly lower parity $(0.6 \pm 0.9$ vs $0.9 \pm 1.1, p<0.001)$ and a higher rate of maternal comorbidities $(17.3 \%$ vs $11.7 \%$, $p=0.002$ ). There were no differences in BMI (Table 1). Of the IVF conception patients, $236(33 \%)$ conceived after IVF with unscreened embryos and $476(67 \%)$ conceived after IVF with PGT-A. Among autologous IVF cycles, there was no difference in patient age between those who underwent PGT-A and those who did not. Patients who conceived after IVF with unscreened embryos were more likely to have conceived after oocyte donation $(28.4 \%$ vs $13.9 \%, p<0.001)$. The average age of the egg donor was also younger in IVF with the unscreened embryo group ( $26.7 \pm 4.8$ years vs $28.6 \pm 5.7$ years, $p$ $=0.038$ ). BMI, parity, rate of comorbidities, and use of gestational carrier did not differ between the two groups (Table 2).

Table 1 Patient demographics - natural conception vs IVF

\begin{tabular}{llll}
\hline & Natural conception $(n=712)$ & $\operatorname{IVF}(n=712)$ & $p$ value \\
\hline Age & $35.0 \pm 5.4$ & $35.0 \pm 5.4$ & $1^{\mathrm{a}}$ \\
Parity & $0.9 \pm 1.1$ & $0.6 \pm 0.9$ & $<0.001^{\mathrm{a}}$ \\
BMI & $24.1 \pm 4.4$ & $24.3 \pm 3.9$ & $0.515^{\mathrm{a}}$ \\
Comorbidities $^{\mathrm{c}}$ & $83(11.7 \%)$ & $123(17.3 \%)$ & $0.002^{\mathrm{b}}$ \\
\hline
\end{tabular}

Data presented as mean \pm standard deviation for parametric continuous variables and $n(\%)$ for categorical variables

${ }^{\mathrm{a}} t$ test

${ }^{\mathrm{b}}$ Chi-square test

${ }^{\mathrm{c}}$ Characterization of comorbidities is detailed in Supplemental Table 1
There were no significant differences in the rate of abnormal anatomy ultrasound, detection of major or minor fetal anomalies, or rate of high-risk NT in patients who underwent IVF with PGT-A compared to patients who conceived following IVF with unscreened embryos and age-matched patients with natural conceptions. The characterization of major and minor anomalies by group is shown in Table 4. Several fetuses with anomalous findings on ultrasound had abnormalities of multiple systems. Table 3 presents the rates of anomalous fetuses per group on a per-patient basis, while Table 4 displays the total number of major and minor anomalies per group. Patients with natural conceptions had a significantly lower rate of abnormal placental findings compared to patients who conceived after IVF with unscreened embryos or IVF with PGT-A (2.9\% vs $8.9 \%$ vs $8.8 \%, p<0.001$ ) (Table 3). This finding of an increased rate of placental abnormalities in patients who conceived after IVF with or without PGT-A when compared to natural conceptions persisted in a multivariate logistic regression model adjusting for the presence of medical comorbidities in the mother $(p<$ $0.001)$. The breakdown in frequency of various placental anomalies is available in Supplemental Table 2. There was no difference in rate of placental anomalies between oocyte donor and autologous IVF pregnancies.

The rate of high-risk NIPT did not differ among patients who underwent IVF with PGT-A compared to patients who conceived following IVF with unscreened embryos and age-matched patients with natural conceptions. Compared to patients who conceived after IVF with unscreened embryos and IVF with PGT-A, patients with natural conceptions had a lower rate of abnormal second trimester serum analytes $(0.9 \%$ vs $3.5 \%$ vs $4.5 \%, p=0.003)$. This finding of a difference in rate of abnormal second trimester screen between treatment groups persisted in a multivariate logistic regression adjusting for the presence of medical comorbidities in the mother $(p=0.002)$. A total of 131 patients elected to undergo invasive diagnostic testing via amniocentesis/CVS. The rate of abnormal invasive diagnostic testing was significantly higher in patients with natural conceptions compared to patients who conceived after IVF with unscreened embryos or IVF with PGT-A $(12.0 \%$ vs $5.9 \%$ vs $0 \%, p=0.049)$ (Table 5). Genetic abnormalities included segmental and whole chromosome mosaicism, microdeletions or microduplications, whole chromosome aneuploidy, and 47 XXX. Notably, there was $100 \%$ concordance of PGT-A with normal karyotype on invasive diagnostic testing though the number of patients who pursued invasive testing was low overall.

\section{Discussion}

In this large cohort, the rate of fetal anomalies detected on mid-trimester ultrasound did not differ in patients who 
Table 2 Patient demographicsIVF no PGT vs IVF + PGT

\begin{tabular}{llll}
\hline & IVF no PGT $(n=237)$ & IVF + PGT $(n=475)$ & $p$ value \\
\hline Age (autologous cycles) & $36.5 \pm 3.9$ & $36.7 \pm 3.8$ & $0.696^{\mathrm{a}}$ \\
Age (oocyte donation cycles) & $26.7 \pm 4.8(n=67)$ & $28.6 \pm 5.7(n=66)$ & $0.038^{\mathrm{a}}$ \\
Parity & $0.6 \pm 0.8$ & $0.6 \pm 0.9$ & $0.672^{\mathrm{b}}$ \\
BMI & $24.7 \pm 4.3$ & $24.1 \pm 3.8$ & $0.053^{\mathrm{a}}$ \\
Comorbidities & $45(19.0 \%)$ & $78(16.4 \%)$ & $0.380^{\mathrm{b}}$ \\
Gestational carrier & $14(5.9 \%)$ & $25(5.3 \%)$ & $0.707^{\mathrm{b}}$ \\
\hline
\end{tabular}

Data presented as mean \pm standard deviation for parametric continuous variables and $n(\%)$ for categorical variables

${ }^{\mathrm{a}} t$ test

${ }^{\mathrm{b}}$ Chi-square test

conceived after IVF with PGT-A compared to patients who conceived following IVF with unscreened embryos and agematched patients with natural conceptions. Rates of abnormal NT and NIPT were also similar between groups. These findings are consistent with prior studies that have reported similar rates of congenital anomalies in neonates conceived through IVF with PGT compared to those conceived with unscreened embryos [15, 20-23]. However, nearly all prior studies involved cleavage stage or polar body biopsy for PGD with FISH or array comparative genome hybridization. While we do not have data on the biopsy technique or testing platform, our cohort was derived from 2017 to 2018, at which time blastocyst stage trophectoderm biopsy with next-generation sequencing was overwhelmingly adopted $[6,24,25]$.
A significantly higher rate of abnormal placental ultrasound findings and abnormal second trimester serum analytes were seen in patients who conceived following IVF with or without PGT-A compared to natural conception controls. Placental complications such as placental insufficiency and preeclampsia have been associated with alterations in second trimester maternal serum analytes, and patients with subfertility who conceive with and without IVF have been demonstrated to have higher rates of abnormal placentation in several large studies [26-30]. The increased rates of abnormal second trimester serum analytes and abnormal placental appearance on mid-trimester ultrasound among patients who conceived after IVF in our study are therefore consistent with prior research. The frequency of detection of subgroups of
Table 4 Fetal anomaly characterization

\begin{tabular}{lllll}
\hline & Natural conception & IVF no PGT & IVF + PGT & $p$ value \\
\hline Major anomalies & $27(3.8 \%)$ & $10(4.2 \%)$ & $18(3.8 \%)$ & $0.948^{\mathrm{a}}$ \\
Nervous & 5 & 0 & 2 & \\
Cardiac & 6 & 7 & 8 \\
GI & 1 & 0 & 1 & \\
GU & 5 & 1 & 1 & \\
MSK/bone & 4 & 1 & 3 \\
Cleft lip/palate & 1 & 0 & 0 & $0.494^{\mathrm{a}}$ \\
Metabolic & 0 & 0 & 2 & \\
Malformation syndrome & 1 & 1 & 2 & \\
Other & 4 & 0 & $58(12.2 \%)$ \\
Minor anomalies & $72(10.1 \%)$ & $28(11.9 \%)$ & 3 \\
Nervous & 13 & 1 & 24 \\
Cardiac & 31 & 17 & 7 & \\
GI & 12 & 3 & 24 & \\
GU & 20 & 6 & 4 & 11 \\
Other & 6 & & \\
\hline
\end{tabular}

Data presented $n(\%)$

GI gastrointestinal, GU genitourinary, MSK musculoskeletal

${ }^{\mathrm{a}}$ Chi-square test 
Table 3 Sonographic evaluation

\begin{tabular}{lllll}
\hline & Natural conception & IVF no PGT & IVF + PGT & $p$ value \\
\hline High-risk NT & $2 / 513(0.4 \%)$ & $2 / 175(1.1 \%)$ & $3 / 371(0.8 \%)$ & $0.350^{\mathrm{b}}$ \\
Anatomy US abnormal & $77 / 712(10.8 \%)$ & $36 / 236(15.2 \%)$ & $68 / 475(14.3 \%)$ & $0.093^{\mathrm{a}}$ \\
Placenta US abnormal & $21 / 712(3.0 \%)$ & $21 / 236(8.9 \%)$ & $42 / 475(8.8 \%)$ & $<0.001^{\mathrm{a}}$ \\
\hline
\end{tabular}

Data presented $n(\%)$, with denominators provided when data is missing

${ }^{\mathrm{a}}$ Chi-square test

${ }^{\mathrm{b}}$ Fisher's exact test

placental abnormalities was small and, therefore, meaningful comparisons could not be made for specific groups of placental abnormalities. This should be investigated further. We did not, however, see a difference in placental abnormalities nor abnormal serum analytes between IVF and IVF with PGT-A. This is consistent with a recent retrospective cohort study which found no difference in rate of abnormal placentation in patients who conceived after IVF with or without PGT-A [31].

The rate of abnormal invasive diagnostic testing was significantly higher among patients with natural conceptions compared to patients who conceived following IVF with or without PGT-A, with a $p$ value that just met significance, $p=$ 0.049 . This difference was driven by a $0 \%$ abnormal invasive testing rate in the IVF with the PGT-A group, who accounted 39 of the 56 IVF patients who underwent invasive testing. This result was not surprising given that PGT-A would be expected to reduce the number of chromosomal abnormalities detectable on CVS/amniocentesis karyotype. While discordance between PGT-A and invasive diagnostic testing may occur in rare cases, we would not expect to see it in our cohort given the sample size.

Previous studies have shown that patients have misconceptions about the utility of PGT-A and that this impacts decisionmaking regarding prenatal care. In a prior publication by one of the authors, the majority of patients undergoing IVF believed PGT-A would improve the chance of having a "healthy baby" and reduce the risk of birth defects [7]. A study evaluating the utilization of prenatal genetic screening among patients at an academic reproductive endocrinology and infertility (REI) clinic found that patients who conceived after IVF with PGT-A were more likely to decline all prenatal screening and diagnostic testing than patients who conceived with unscreened embryos, $19.1 \%$ vs $4 \%$ respectively [32]. The results of the present study provide evidence that PGT-A does not preclude or even significantly reduce the risk of fetal anomalies in pregnancies conceived with IVF. This highlights the importance of improved patient counseling regarding the limitations of PGT-A, and of providing standard prenatal care for pregnancies conceived through ART, including prenatal aneuploidy screening and diagnostic testing, regardless of whether PGT was performed.

Limitations of this study include its retrospective design and the potential for selection bias given that the study setting is a high-risk referral center. The rates of anomalies in this patient population, therefore, may not reflect those in the general population. We aimed to mitigate this by inclusion of a natural conception control group in which we found a baseline incidence of anomalies similar to what has previously reported in the general population. We did not include birth outcome, newborn exam data, or placental pathology data and therefore could not corroborate the anomalies detected on mid-trimester ultrasound with clinical outcomes. While this is a limitation, it is worth noting that many pregnancies complicated by major anomalies detected on mid-trimester ultrasound are terminated and, thus, would not necessarily be captured by inclusion of newborn examination data. Finally, because only women who underwent mid-trimester ultrasound were included, this would not capture early miscarriages or terminations.

Strengths of our study include the large sample size, inclusion of a natural conception control group, and that all analyzed data originated from 2017 to 2018, which increases the likelihood that IVF and PGT-A technologies used were more homogenous. Additionally, to our knowledge, this is the first
Table 5 Serum screening and invasive diagnostic testing

\begin{tabular}{lllll}
\hline & Natural conception & IVF no PGT & IVF + PGT & $p$ value $^{a^{a}}$ \\
\hline NIPT high-risk & $9 / 622(1.5 \%)$ & $1 / 186(0.5 \%)$ & $3 / 395(0.8 \%)$ & 0.550 \\
Second trimester serum analyte abnormal & $5 / 537(0.9 \%)$ & $6 / 172(3.5 \%)$ & $16 / 358(4.5 \%)$ & 0.002 \\
Invasive diagnostic testing abnormal & $9 / 75(12.0 \%)$ & $1 / 17(5.9 \%)$ & $0 / 39(0 \%)$ & 0.049 \\
\hline
\end{tabular}

Data presented as $n(\%)$, with denominators provided when data is missing

${ }^{\text {a }}$ Fisher's exact test 
study to report the rate of ultrasound anomalies, fetal and placental, in IVF patients with and without PGT-A.

In conclusion, the use of PGT-A was not associated with a difference in the rate of fetal anomalies detected on midtrimester ultrasound in this large cohort. While the available evidence does not support the use of PGT-A for the purpose of reducing the risk of birth defects, patients consistently report this as a primary reason for pursuing this technology [7]. Improvement in patient counseling regarding the limitations of PGT-A is crucial.

Supplementary Information The online version contains supplementary material available at https://doi.org/10.1007/s10815-021-02069-5.

Authors' contributions C.R., M.Q., and C.H. participated in study design. C.R., T.M., and J.R.O. participated in data collection. C.R. and M.Q. participated in data analysis. C.R., M.Q., T.M., C.H., and L.P. participated in manuscript drafting and critical discussion.

Data availability Coded data pertaining to this study are stored on a password and firewall protected server and can be made available if needed.

\section{Compliance with ethical standards}

Conflict of interest The authors declare that they have no conflicts of interest.

Ethics approval This study was approved by the Institutional Review Board of the University of California, Los Angeles (IRB\# 19-000222).

Consent to participate Because of the retrospective nature of this study, the UCLA IRB deemed the study to have minimal risk and waived the requirement for informed consent.

Consent for publication Consent to publish was not required as the UCLA IRB deemed this study of minimal risk.

Code availability Code for the data can be made available if required.

Open Access This article is licensed under a Creative Commons Attribution 4.0 International License, which permits use, sharing, adaptation, distribution and reproduction in any medium or format, as long as you give appropriate credit to the original author(s) and the source, provide a link to the Creative Commons licence, and indicate if changes were made. The images or other third party material in this article are included in the article's Creative Commons licence, unless indicated otherwise in a credit line to the material. If material is not included in the article's Creative Commons licence and your intended use is not permitted by statutory regulation or exceeds the permitted use, you will need to obtain permission directly from the copyright holder. To view a copy of this licence, visit http://creativecommons.org/licenses/by/4.0/.

\section{References}

1. SART. Society for Assisted Reproductive Technology [Internet]. SART Clin. Summ. Rep. 2018; 2018. [cited 2020 Jun 10].
Available from: https://www.sartcorsonline.com/rptCSR PublicMultYear.aspx?\#patient-cumulative. Accessed 1 Aug 2019.

2. Dahdouh EM, Balayla J, García-Velasco JA. Comprehensive chromosome screening improves embryo selection: a meta-analysis. Fertil Steril. 2015;104:1503-12.

3. Forman EJ, Tao X, Ferry KM, Taylor D, Treff NR, Scott RT. Single embryo transfer with comprehensive chromosome screening results in improved ongoing pregnancy rates and decreased miscarriage rates. Hum Reprod. 2012;27:1217-22.

4. Yang Z, Liu J, Collins GS, Salem SA, Liu X, Lyle SS, et al. Selection of single blastocysts for fresh transfer via standard morphology assessment alone and with array CGH for good prognosis IVF patients: results from a randomized pilot study. Mol Cytogenet. 2012;5:24.

5. Scott RT, Upham KM, Forman EJ, Hong KH, Scott KL, Taylor D, et al. Blastocyst biopsy with comprehensive chromosome screening and fresh embryo transfer significantly increases in vitro fertilization implantation and delivery rates: a randomized controlled trial. Fertil Steril. 2013;100:697-703.

6. Munné S, Kaplan B, Frattarelli JL, Child T, Nakhuda G, Shamma FN, et al. Preimplantation genetic testing for aneuploidy versus morphology as selection criteria for single frozen-thawed embryo transfer in good-prognosis patients: a multicenter randomized clinical trial. Fertil Steril. 2019;112:1071-9.

7. Quinn MM, Juarez-Hernandez F, Dunn M, Okamura RJ, Cedars MI, Rosen MP. Decision-making surrounding the use of preimplantation genetic testing for aneuploidy reveals misunderstanding regarding its benefit. J Assist Reprod Genet. 2018;35:2155-9.

8. Boyd PA, Rounding C, Chamberlain P, Wellesley D, Kurinczuk JJ. The evolution of prenatal screening and diagnosis and its impact on an unselected population over an 18-year period. BJOG. 2012;119: 1131-40.

9. Anasti JN, Flack MR, Froehlich J, Nelson LM. The use of human recombinant gonadotropin receptors to search for immunoglobulin G-mediated premature ovarian failure. J Clin Endocrinol Metab. 1995;80(3):824-8. https://doi.org/10.1210/jcem.80.3.7883837.

10. Brent RL. Environmental causes of human congenital malformations: the pediatrician's role in dealing with these complex clinical problems caused by a multiplicity of environmental and genetic factors. Pediatrics. 2004;113(4 Suppl):957-68.

11. Cash C, Set P, Coleman N. The accuracy of antenatal ultrasound in the detection of facial clefts in a low-risk screening population. Ultrasound Obstet Gynecol. 2001;18:432-6.

12. Barisic I, Clementi M, Husler M, Gjergja R, Kern J, Stoll C. Evaluation of prenatal ultrasound diagnosis of fetal abdominal wall defects by 19 European registries. Ultrasound Obstet Gynecol. 2001;18:309-16.

13. Chew C, Halliday JL, Riley MM, Penny DJ. Population-based study of antenatal detection of congenital heart disease by ultrasound examination. Ultrasound Obstet Gynecol. 2007;29:619-24.

14. Schechtman KB, Gray DL, Baty JD, Rothman SM. Decisionmaking for termination of pregnancies with fetal anomalies: analysis of 53,000 pregnancies. Obstet Gynecol. 2002;99:216-22.

15. Zhang WY, von Versen-Höynck F, Kapphahn KI, Fleischmann RR, Zhao Q, Baker VL. Maternal and neonatal outcomes associated with trophectoderm biopsy. Fertil Steril. 2019;112:283-90.

16. CDC - Centers for Desease Control And Prevention. Timeline ADHD $\mid$ NCBDDD | CDC. Div Hum Dev Disabil Natl Cent Birth Defects Dev Disabil Centers Dis Control Prev [Internet]. 2017; Available from: https://www.cdc.gov/ncbddd/birthdefects/ surveillancemanual/chapters/chapter-1/chapter1-4.html. Accessed 1 Aug 2019.

17. Nicolaides KH, Azar G, Byrne D, Mansur C, Marks K. Fetal nuchal translucency: ultrasound screening for chromosomal defects in first trimester of pregnancy. Br Med J. 1992;304:867-9. https://doi.org/ 10.1136/bmj.304.6831.867. 
18. Wald NJ, Rodeck C, Hackshaw AK, Walters J, Chitty L, Mackinson AM. First and second trimester antenatal screening for Down's syndrome: the results of the Serum, Urine and Ultrasound Screening Study (SURUSS). J Med Screen. 2003;10(2):56-104.

19. Malone FD, Canick JA, Ball RH, Nyberg DA, Comstock CH, Bukowski R, et al. First-trimester or second-trimester screening, or both, for down's syndrome. N Engl J Med. 2005;353:2001-11.

20. Bay B, Ingerslev HJ, Lemmen JG, Degn B, Rasmussen IA, Kesmodel US. Preimplantation genetic diagnosis: a national multicenter obstetric and neonatal follow-up study. Fertil Steril. 2016;106:1363-9.

21. Forman EJ, Hong KH, Franasiak JM, Scott RT. Obstetrical and neonatal outcomes from the BEST Trial: single embryo transfer with aneuploidy screening improves outcomes after in vitro fertilization without compromising delivery rates. Am J Obstet Gynecol. 2014;210:e1-6.

22. Desmyttere S, De Rycke M, Staessen C, Liebaers I, De Schrijver F, Verpoest W, et al. Neonatal follow-up of 995 consecutively born children after embryo biopsy for PGD. Hum Reprod. 2012;27:28893.

23. Liebaers I, Desmyttere S, Verpoest W, De Rycke M, Staessen C, Sermon K, et al. Report on a consecutive series of 581 children born after blastomere biopsy for preimplantation genetic diagnosis. Hum Reprod. 2010;25:275-82.

24. Treff NR, Scott RT. Four-hour quantitative real-time polymerase chain reaction-based comprehensive chromosome screening and accumulating evidence of accuracy, safety, predictive value, and clinical efficacy. Fertil Steril. 2013;99:1049-53.

25. Friedenthal J, Maxwell SM, Munné S, Kramer Y, McCulloh DH, McCaffrey $\mathrm{C}$, et al. Next generation sequencing for preimplantation genetic screening improves pregnancy outcomes compared with array comparative genomic hybridization in single thawed euploid embryo transfer cycles. Fertil Steril. 2018;109:627-32.

26. Lakhi N, Govind A, Moretti M, Jones J. Maternal serum analytes as markers of adverse obstetric outcome. Obstet Gynaecol. 2012;14: 267-73.

27. Luke B, Gopal D, Cabral H, Stern JE, Diop H. Pregnancy, birth, and infant outcomes by maternal fertility status: the Massachusetts Outcomes Study of Assisted Reproductive Technology. Am J Obstet Gynecol. 2017;217:e1-14.

28. Shevell T, Malone FD, Vidaver J, Porter TF, Luthy DA, Comstock $\mathrm{CH}$, et al. Assisted reproductive technology and pregnancy outcome. Obstet Gynecol. 2005;106:1039-45.

29. Romundstad LB, Romundstad PR, Sunde A, von Düring V, Skjærven R, Vatten LJ. Increased risk of placenta previa in pregnancies following IVF/ICSI; a comparison of ART and non-ART pregnancies in the same mother. Hum Reprod. 2006;21:2353-8.

30. Ebbing C, Kiserud T, Johnsen SL, Albrechtsen S, Rasmussen S. Prevalence, risk factors and outcomes of velamentous and marginal cord insertions: a population-based study of 634,741 pregnancies. PLoS One. 2013;8:e70380.

31. Swanson K, Wijekoon A, Huang D, Rosenstein MG, Blat C, MokLin E, et al. 197: does preimplantation genetic testing increase the risk of abnormal placentation in IVF pregnancies? Am J Obstet Gynecol. 2020;222(1):S136-7.

32. Kimelman D, Confino R, Confino E, Shulman LP, Zhang JX, Pavone ME. Do patients who achieve pregnancy using IVF-PGS do the recommended genetic diagnostic testing in pregnancy? $\mathrm{J}$ Assist Reprod Genet. 2018;35:1881-5.

Publisher's note Springer Nature remains neutral with regard to jurisdictional claims in published maps and institutional affiliations. 